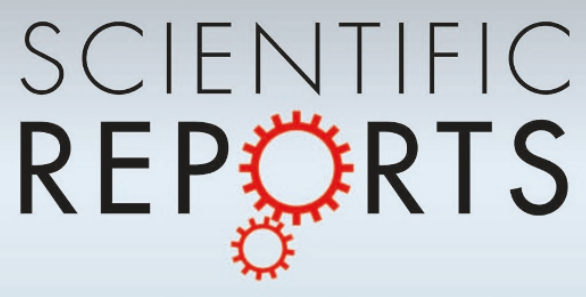

\title{
OPEN \\ SUBJECT AREAS: \\ EPILEPSY \\ High dose folic acid supplementation of rats alters synaptic transmission and seizure susceptibility in offspring
}

SYNAPTIC TRANSMISSION

CELLULAR NEUROSCIENCE

DEVELOPMENT OF THE NERVOUS

SYSTEM

Received

4 December 2012

Accepted

28 February 2013

Published

15 March 2013

Correspondence and requests for materials should be addressed to

M.A.C. (mcolicos@ ucalgary.ca)

* These authors contributed equally to this work.

\author{
Fernando Girotto ${ }^{3 *}$, Lucas Scott ${ }^{*}$, Yosef Avchalumov ${ }^{1}$, Jacqueline Harris ${ }^{1}$, Stephanie lannattone ${ }^{1}$, \\ Chris Drummond-Main ${ }^{2}$, Rose Tobias², Luis Bello-Espinosa², Jong M. Rho², Jörn Davidsen ${ }^{3}$, \\ G. Campbell Teskey ${ }^{4} \&$ Michael A. Colicos ${ }^{1}$
}

'Department of Physiology \& Pharmacology, Faculty of Medicine, and the Hotchkiss Brain Institute, University of Calgary, Calgary, $\mathrm{AB}$, Canada T2N 4N1, ${ }^{2}$ Pediatric Neurology and the Alberta Children's Hospital Research Institute, University of Calgary, Calgary, $\mathrm{AB}$, Canada T2N 4N1, ${ }^{3}$ Complexity Science Group, Department of Physics and Astronomy, Faculty of Science, University of Calgary, Calgary, AB, Canada T2N 4N1, ${ }^{4}$ Department of Cell Biology \& Anatomy, Faculty of Medicine, and the Hotchkiss Brain Institute, University of Calgary, Calgary, AB, Canada T2N 4N1.

Maternal folic acid supplementation is essential to reduce the risk of neural tube defects. We hypothesize that high levels of folic acid throughout gestation may produce neural networks more susceptible to seizure in offspring. We hence administered large doses of folic acid to rats before and during gestation and found their offspring had a $42 \%$ decrease in their seizure threshold. In vitro, acute application of folic acid or its metabolite 4 Hfolate to neurons induced hyper-excitability and bursting. Cultured neuronal networks which develop in the presence of a low concentration $(50 \mathrm{nM})$ of 4 Hfolate had reduced capacity to stabilize their network dynamics after a burst of high-frequency activity, and an increase in the frequency of mEPSCs. Networks reared in the presence of the folic acid metabolite 5M4Hfolate developed a spontaneous, distinctive bursting pattern, and both metabolites produced an increase in synaptic density.

- he discovery that dietary folic acid supplementation before and during pregnancy can dramatically reduce the incidence of neural tube defects has been a major advance for child health ${ }^{1,2}$. However since the neural tube closes at day 27 during human gestation ${ }^{3}$, high levels of folic acid after this point may serve no benefit, and could even be detrimental ${ }^{4}$. Folic acid and its related compounds are based on pteroic acid conjugated to Lglutamate, the latter being the major excitatory neurotransmitter in the brain, and folic acid itself has been previously demonstrated to have excitatory effects ${ }^{5-7}$. As there are glutamatergic synapses present in the hippocampus as early as the 3 rd trimester $^{8}$, and given the tight link between neuronal activity and synaptic development, we hypothesized that the continued presence of folic acid during neuronal development might alter the delicate balance of normal network formation and function.

Our experimental approach was as follows. First we demonstrated that folic acid can directly increase neuronal excitability by acutely applying it to cultured neurons while recording their activity. With this established, we pursued the hypothesis that folic acid exposure increases neuronal activity during development, possibly resulting in abnormal brain development, since many aspects of the establishment of neuronal connectivity are dependent on activity. We tested if administration of folic acid to pregnant rats leads to lowered seizure threshold in offspring, using a kainic acid model of seizure induction. We then used an in vitro primary neuronal culture model to investigate the mechanism in detail, in which we allow neuronal cultures to develop in the presence of low concentrations of 4 Hfolate or $5 \mathrm{M} 4 \mathrm{Hf}$ folate, replicating the in utero conditions. We chose these two metabolites of folic acid for the following reasons. The liver metabolizes folic acid to $4 \mathrm{Hfolate}$, and so for the in vitro study this is the highest-level biologically active form we can use. When added to the media, 4Hfolate will in part be catabolized to pterin and p-aminobenzoylglutamate scission products, the latter of which will go on to produce Lglutamate. Enzymatically, 4Hfolate is also metabolized to 5M4Hfolate, a form commonly studied in terms of its effect on cellular physiology. Therefore in experiments using 4 Hfolate we could determine the full spectrum of effects caused by folic acid, and by adding just 5M4Hfolate, we determine the effect of this specific metabolite alone. Similar to the in vivo experiments, we challenge the 4 Hfolate and 5M4Hfolate reared cultures with a burst of high frequency activity and determine how well they re-stabilized their activity level. Previous reports have 
suggested that folic acid can alter synaptic density ${ }^{9}$, and so we look for structural changes in connectivity, using the distribution patterns of the synaptic maker synaptophysin. Finally, we analyze miniature EPSCs from neurons grown in the presence of $4 \mathrm{Hfolate}$ and demonstrate an increase in the frequency of the mEPSC events. We hypothesize that folic acid supplementation enhances neuronal activity and consequently the rate of development, potentially resulting in hyper-excitability and ultimately may lead to a network that is more vulnerable to seizures.

\section{Results}

Folic acid and neuronal excitability. Based on studies from the mid 1980 's ${ }^{5,6}$, we hypothesized that exposing neurons to folic acid would alter their firing rate. Current clamp recordings were made from $\sim 3$ weeks in vitro neurons in dissociated hippocampal culture networks, to which either folic acid, 4 Hfolate or vehicle alone was acutely added. As previously stated, 4 Hfolate was investigated since folic acid is first metabolized in the liver to this active form ${ }^{10}$. When $10 \mathrm{mM}$ concentrations of folic acid or 4 Hfolate were applied to the neuron, a distinct change in the firing pattern of the cell was observed. In neurons which did not show a bursting pattern of activity during the initial recording period, both folic acid and 4 Hfolate caused them rapidly (within minutes) to fire in bursts, whereas this was not observed with the controls (Figure 1a,b,c). This effect was protracted, being observable at least 10 minutes following this initial application of the drug (shown for folic acid in Figure 1D). These results suggest that folic acid and its derivatives have the potential acutely to alter the firing rate of individual neurons, and consequently alter the firing dynamics of a neuronal system.

Maternal exposure to dietary folic acid during gestation. The observation that folic acid and its derivatives could alter neuronal firing raised the possibility that its presence during gestation may affect development. To examine the potential in vivo relevance of this we tested the hypothesis that maternal exposure to dietary folic acid during gestation may make the offspring more vulnerable to a challenge by a convulsant drug. Pups from dams that received folic acid (4 mg administered in $1 \mathrm{ml}$ of $10 \%$ sucrose) had significantly $(\mathrm{t}(9,8)=2.76, \mathrm{p}=0.02)$ shorter mean seizure onset times $(470$ seconds) following administration of kainic acid (KA) relative to the control treated pups (813 seconds). This directly demonstrates that the presence of dietary folic acid leads to an almost 2 fold increase in seizure susceptibility (Figure 2f).

Analysis of network activity in $\mathbf{4 H f o l a t e}$ reared cultures. We sought to determine if the presence of folic acid during the developmental establishment of neuronal connectivity affects the nature of the resulting network formed. To do this we grew neuronal networks from dissociated hippocampi from P0 rat pups (see methods) in media supplemented with low levels of 4 Hfolate and characterized the activity patterns of the mature circuits. While several timepoints were tested, we chose 3 week old cultures as this amount of time allows the activity patterns to mature and become more consistent. Analysis of the firing events produces "fingerprints" of neuronal activity as illustrated in Figure $2 \mathrm{a}-\mathrm{d}$, where each individual neuron's activity is represented on one horizontal line over time. A tick mark is added to the plot when a neuron fires. To demonstrate the effectiveness of this method, we monitored culture-wide activity during direct application of bath solution alone (Figure 2a) or 4Hfolate (Figure 2b). These fingerprints show how increased activity (dark vertical band after addition of 4 Hfolate) and bursting (vertical bands of synchronous firing of the population of neurons several minutes after the addition of 4 Hfolate) can be easily visualized in the entire network. To assay the cultures that had been reared in $4 \mathrm{Hf}$ folate, baseline activity was first recorded for two minutes, then all of the neurons in the field of view were stimulated
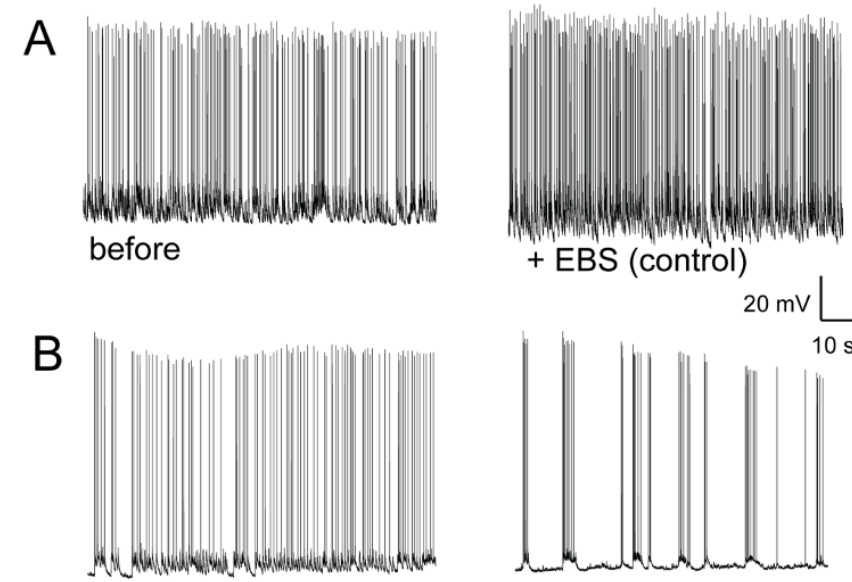

before
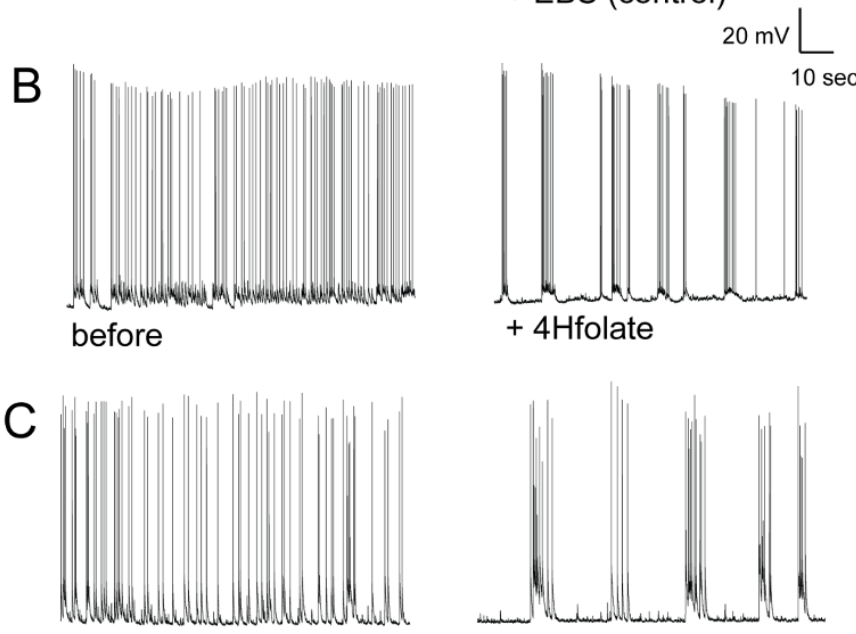

before

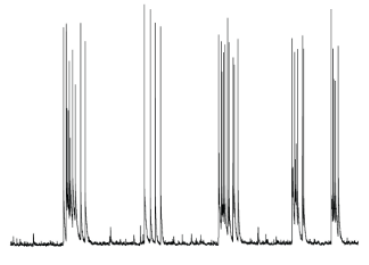

+ folic acid

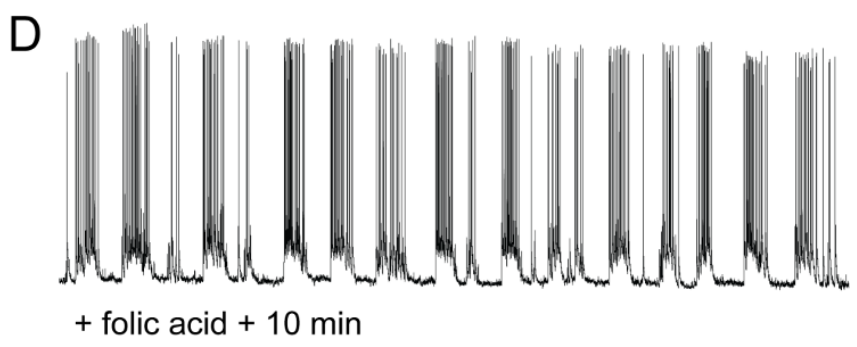

Figure 1 Direct application of $4 \mathrm{Hfolate}$ and folic acid on hippocampal neurons. Current clamp recordings from $\sim 3$ week old dissociated cultures of rat hippocampal neurons. Before tracings show representative cells before treatment. (A) Control experiment, where bath solution alone was spritzed on the neuron. No significant change in the firing patterns was observed $(n=9)$. (B) Recording taken immediately after directly applying $3 \mu \mathrm{l}$ of 4 Hfolate $(10 \mathrm{mM})$ suspended in bath solution on the neuron. Cells rapidly assume a bursting pattern $(n=9)$. (C) Similar results were

obtained using $3 \mu \mathrm{l}$ of $10 \mathrm{mM}$ folic acid. (D) Recording taken 10 minutes after addition of folic acid in $(\mathrm{C})$, at which point the bath concentration is $10 \mu \mathrm{M}$ folic acid. The distinctive change in the firing pattern of the neuron is maintained $(n=9)$. Scale bars in (A) apply to (A-D).

for 30 seconds using photoconductive stimulation (indicated by the red strip on the fingerprints). We continued to record activity for three minutes following the stimulus. Figure $2 c$ shows that control cultures had a healthy level of stochastic spontaneous activity prior to the stimulation, and afterward there was an overall reversion to previous activity levels. In contrast, cultures grown in the presence of $4 \mathrm{Hfolate}$ (Figure $2 \mathrm{~d}$ ) had an increase $(\sim 2.5$ fold, Figure $2 \mathrm{e})$ in the overall level of activity $(n=17, p<0.05)$ following stimulation. Since network activity patterns in dissociated cultures can be variable, samples were taken from multiple regions of each chip, from five separate culture rounds, and experiments were performed over the course of eight months to control for the potential of seasonal variation, none of which was observed.

Analysis of network activity in 5M4Hfolate reared cultures. As stated above, one of the metabolites of $4 \mathrm{Hfolate}$ is $5 \mathrm{M} 4 \mathrm{Hf}$ folate, a 

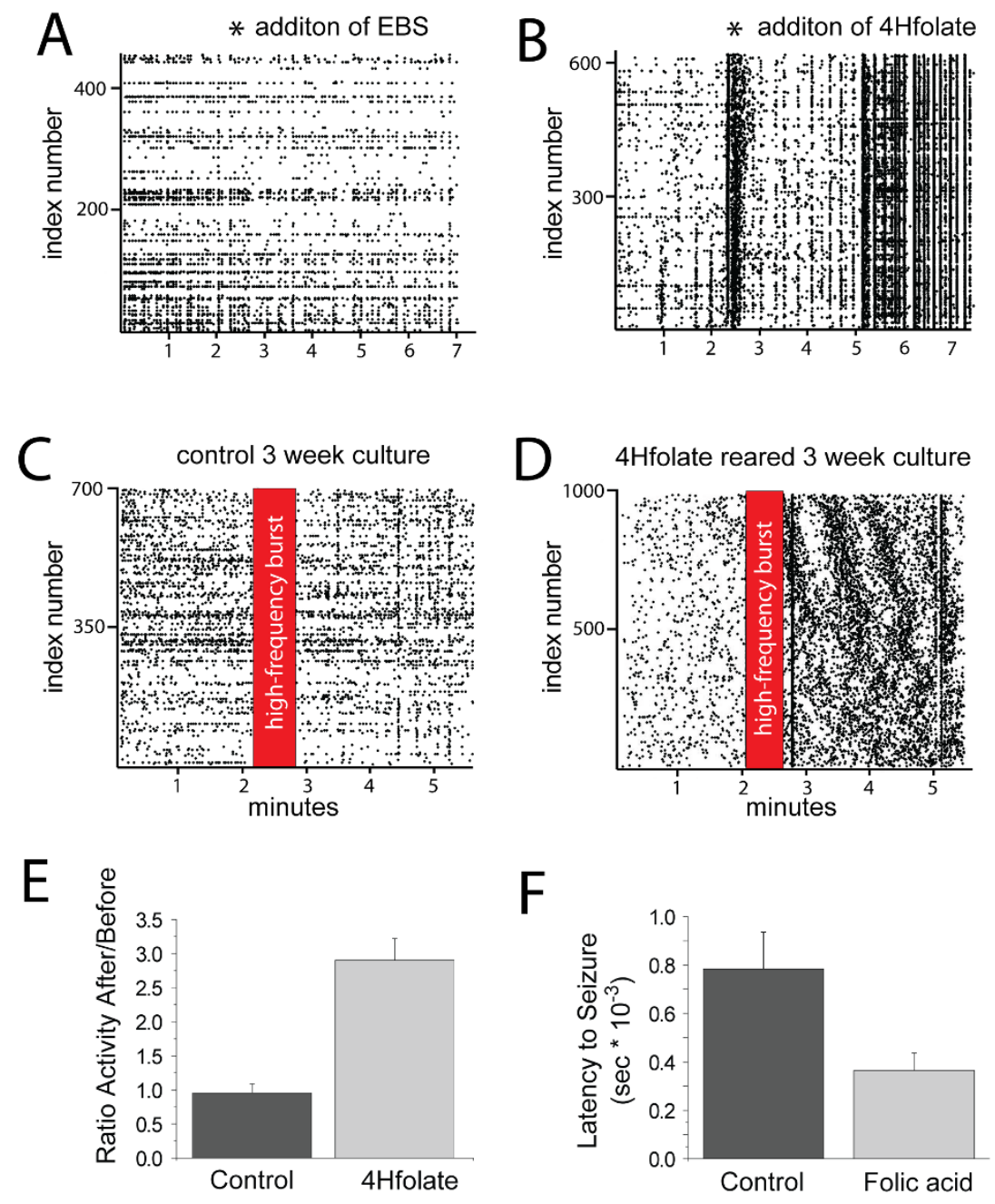

Figure $2 \mid$ In vitro and in vivo effect of folic acid on neuronal activity (A-D). Dissociated co-cultures of neurons and glia were grown on silicon wafers, stained with Fluo-4 and the activity recorded. Firing events in each of up to 1000 neurons (cell index number on vertical axis) are recorded, detected with software, and represented by a tick mark. (A) Direct application of extracellular buffer had no substantial effect on the network activity of 3 week old cultures. (B) Similar to the electrophysiological recordings in Figure 1, optical recording from 1000 neurons show the entire network responding to the acute addition of $4 \mathrm{Hfolate}$ (C) and (D) show the effect of rearing cultures in the presence of low levels (50 $\mathrm{nM}$ ) of $4 \mathrm{Hfolate}$ in comparison to controls. Stochastic activity during a 2 minute control period is recorded, followed by a $30 \mathrm{sec}$ stimulation of all neurons at $60 \mathrm{~Hz}$ (red stripe), and then 3 minutes post-stimulation. (C) Control cultures reverted rapidly to pre-stimulation levels of activity. (D) Culture grown in the presence of $4 \mathrm{Hfolate}$ for 3 weeks prior to testing, showed a continued increase in activity following stimulation. (E) Statistics of the ratio of overall firing rate of the culture after/ before stimulation. Statistics are from $n=14$ recordings for each condition, taken from 5 separate culture rounds over the course of 4 months.

(F) Results from the in vivo study shown for comparison, showing the increased sensitivity (a decrease in the latency to seizure) of pups from dams supplemented with $4.0 \mathrm{mg}$ of folic acid in $1 \mathrm{ml}$ of sucrose daily (control: $813 \mathrm{sec}$, folic acid: $469 \mathrm{sec}$ ). All values are significantly different at $\mathrm{p}<0.05$ (t-test).

form commonly associated with regulation of cellular functions, and it is linked to a variety of disorders ${ }^{11-13}$, including epilepsy ${ }^{14}$. While the breakdown of 4 Hfolate to L-glutamate could be a major component of the observed effects, we also tested 5M4Hfolate in the in vitro assay system. The presence of $5 \mathrm{M} 4 \mathrm{Hf}$ folate during culture development had an intriguing effect. Figure 3a,b shows a comparison between a control and a 5M4Hfolate reared culture. While a less dramatic overall enhancement of network activity persisted after stimulation in comparison to 4 Hfolate, a distinctive pattern of cell firing was observed. A subpopulation of neurons was seen to fire rapidly for short periods of time (Figure $3 \mathrm{~b}$ ). To quantify this effect we calculated the time intervals between firing events and this data is displayed as a histogram in Figure $3 \mathrm{c}, \mathrm{d}$. While the control cultures generated a fairly stochastic distribution of inter-event timings, the 5M4Hfolate reared cultures showed a radically different pattern, with the repeated burst frequencies giving a distinctive population of inter-event times. This pattern was seen in many cultures even in the absence of stimulation ( $n=6 / 8$, Figure $3 e$ ), and was quantified in terms of their order (entropy) in comparison to controls (Figure $3 \mathrm{f}$ ). This result suggests that there is a decrease in entropy, or an increase in order, in the firing events in the $5 \mathrm{M} 4$ Hfolate reared cultures.

Together, the 4 Hfolate and the 5M4Hfolate data demonstrate that there are quantitative differences in response to a high-frequency burst of activity between control and folate reared cultures. Control cultures appear to have the ability to maintain their level and pattern of firing rates following a stimulation event, whereas neuronal networks formed in the presence of folate tend to lose this firing rate pattern or the ability to regulate their firing level. This finding can be compared to the in vivo data, which shows the $\sim 2$ fold increase in susceptibility to seizure, presented graphically in Figure 2f. Furthermore, there is a qualitative difference between 
A

control 2 week culture

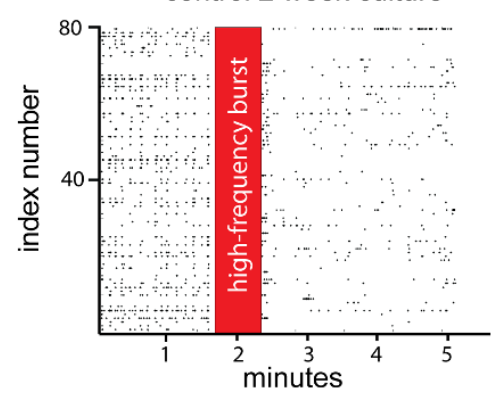

C

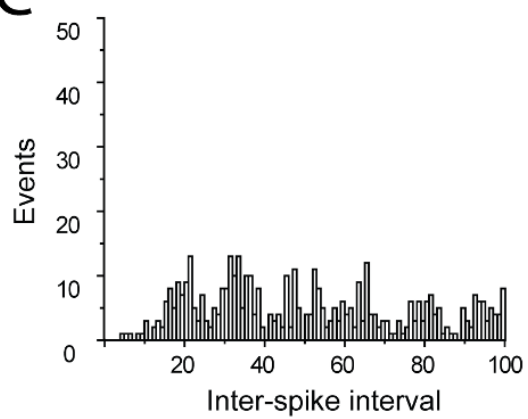

E

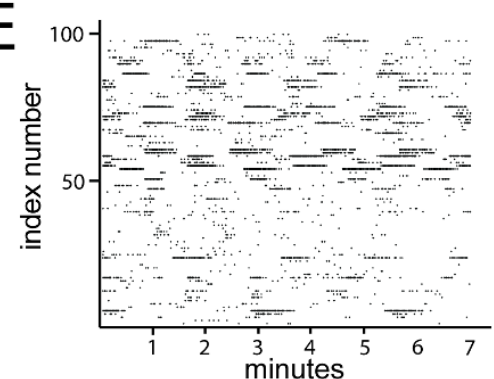

B

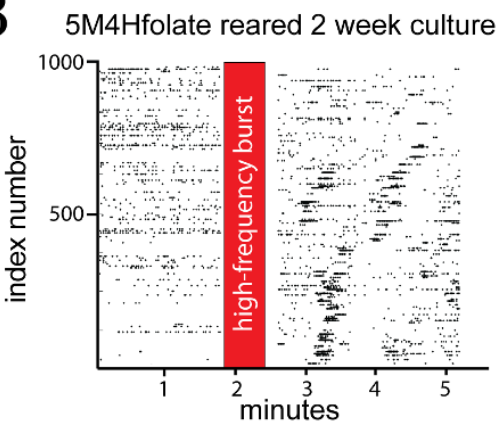

D

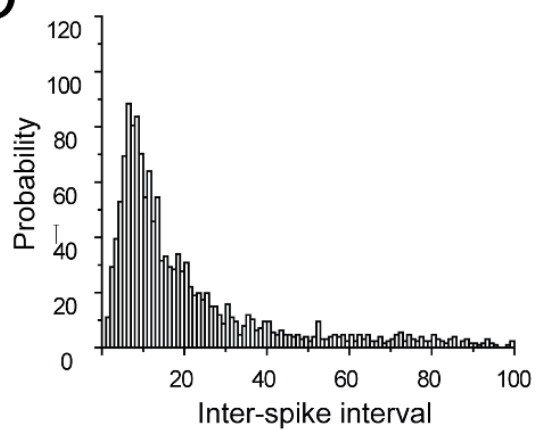

$\mathrm{F}$

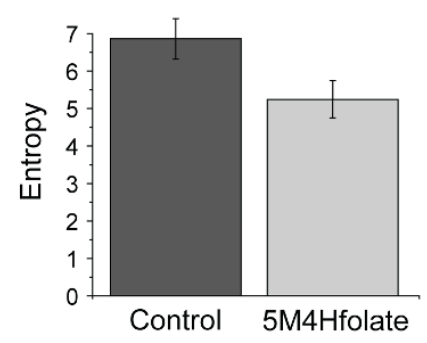

Figure 3 Activity patterns of 5M4Hfolate reared cultures. Activity patterns recorded from 2 week old hippocampal cultures from (A) control and (B) 5M4Hfolate reared conditions challenged with a high frequency burst of activity. While some loss of stability was observed in the 5M4Hfolate cultures, more notable was the unique hyperexcitability of a subset of the neurons (horizontal streaks). To quantify this for controls and 5M4Hfolate we calculated the interval between firing events and plotted their distribution as a histogram in (C), and (D) respectively. It can be seen that while the control cultures have a relatively stochastic distribution of event timings, the $5 \mathrm{M} 4 \mathrm{Hfolate}$ reared cultures have a much more specific inter-event timing. Moreover, this unique pattern could be often observed in unstimulated cultures ( $\mathrm{N}=6$ of 8 cultures tested, (E)). To quantitatively compare the order in these patterns entropy ${ }^{22}$ was calculated (from $\mathrm{N}=4$ cultures) and is shown in figure (F).

neuronal networks reared with each folic acid metabolites, suggesting that each may have a different effect on the connectivity and consequently the nature of the excitability.

Folate reared cultures have increased synaptic density. After identifying a difference in the network activity between control, 4 Hfolate and 5M4Hfolate reared cultures, we subsequently did a structural comparison between the conditions to see if there were any overt changes in synaptic distribution. Cultures used for the activity movies were fixed and immunostained with an antibody against synaptophysin (Figure $4 \mathrm{a}-\mathrm{f}$ ). We found that there was a significant increase in synaptic density in both the $4 \mathrm{Hf}$ folate and the 5M4Hfolate cultures after 1 week of growth, however this quantitative difference diminished after 2 weeks (Figure $4 \mathrm{~g}, \mathrm{~h}$ ). Interestingly there was a qualitative change in the folate reared cultures at two weeks. While the control cultures appeared to have a relatively homogeneous distribution (Figure $4 \mathrm{~d}$ ), the folate reared cultures showed a distinct patterning in their synaptic distribution along neuronal processes (Figure 4e,f). While it is tempting to interpret this as an effect of the higher activity levels inducing synaptic pruning and organization, further studies are being pursued to more accurately quantify this phenomenon.
Rearing in the presence of $4 \mathrm{Hfolate}$ alters neuronal electrophysiology. The differences in basic structural connectivity at a gross level prompted us to investigate whether synaptic transmission was altered in $4 \mathrm{Hf}$ folate reared cultures. We recorded mEPSCs from both cultures (Figure 5a,b), and observed differences in frequency of the events (Figure 5b,c), however there was no significant change in amplitude (Figure 5d,e). This result correlates with the enhanced synaptic density seen in the folate reared cultures during the initial stages of network development. However there is also an increase in mEPSC frequency at 4 weeks, yet only qualitative differences in synaptic distribution at the later timepoint. This suggests other mechanisms might be at work modifying activity in more mature cultures.

\section{Discussion}

Folic acid supplementation during the first month of fetal development is critical to ensure the closure of the neural tube, however the necessity to maintain high folate levels after this event has not been substantiated. As an initial step toward investigating the underlying physiology of folic acid's effect on brain development, we used both in vivo and in vitro rodent paradigms. In an in vivo latency-to-seizure 

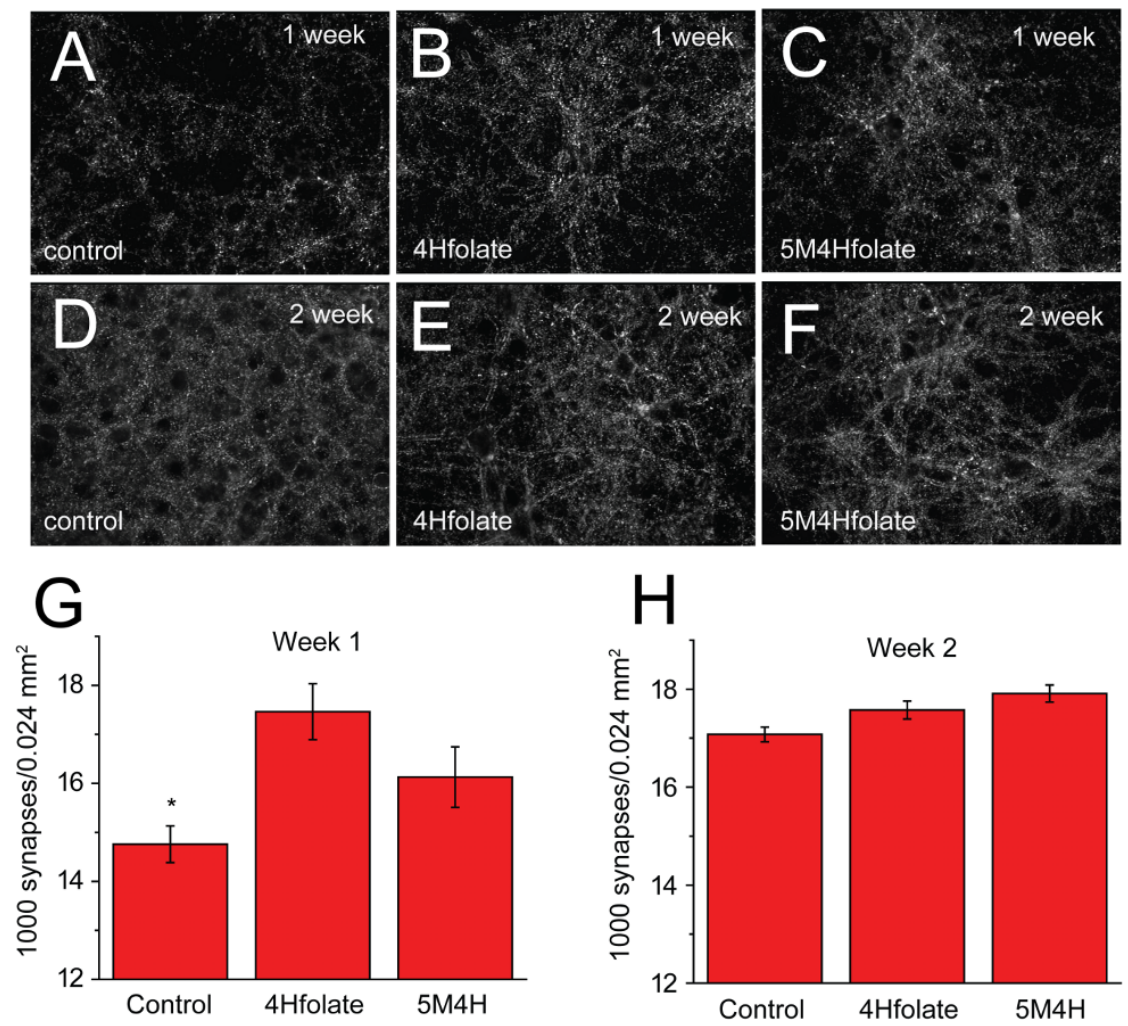

Figure $4 \mid$ Analysis of synaptic distribution in control and folate reared cultures. 1 and 2 week old (A,D) control, (B,E) 4Hfolate reared and (C,F) $5 \mathrm{M} 4 \mathrm{Hf}$ folate reared cultures were immunostained for synaptophysin. Synaptic counts were extracted from 8 regions from each culture, and 3 separate culture rounds were analyzed. Statistics for $(\mathrm{G}) 1$ week and $(\mathrm{H}) 2$ week counts, showing controls significantly lower at one week from both metabolites $(\mathrm{n}=24, \mathrm{p}<0.05$ (t-test) $)$

\section{A}

control
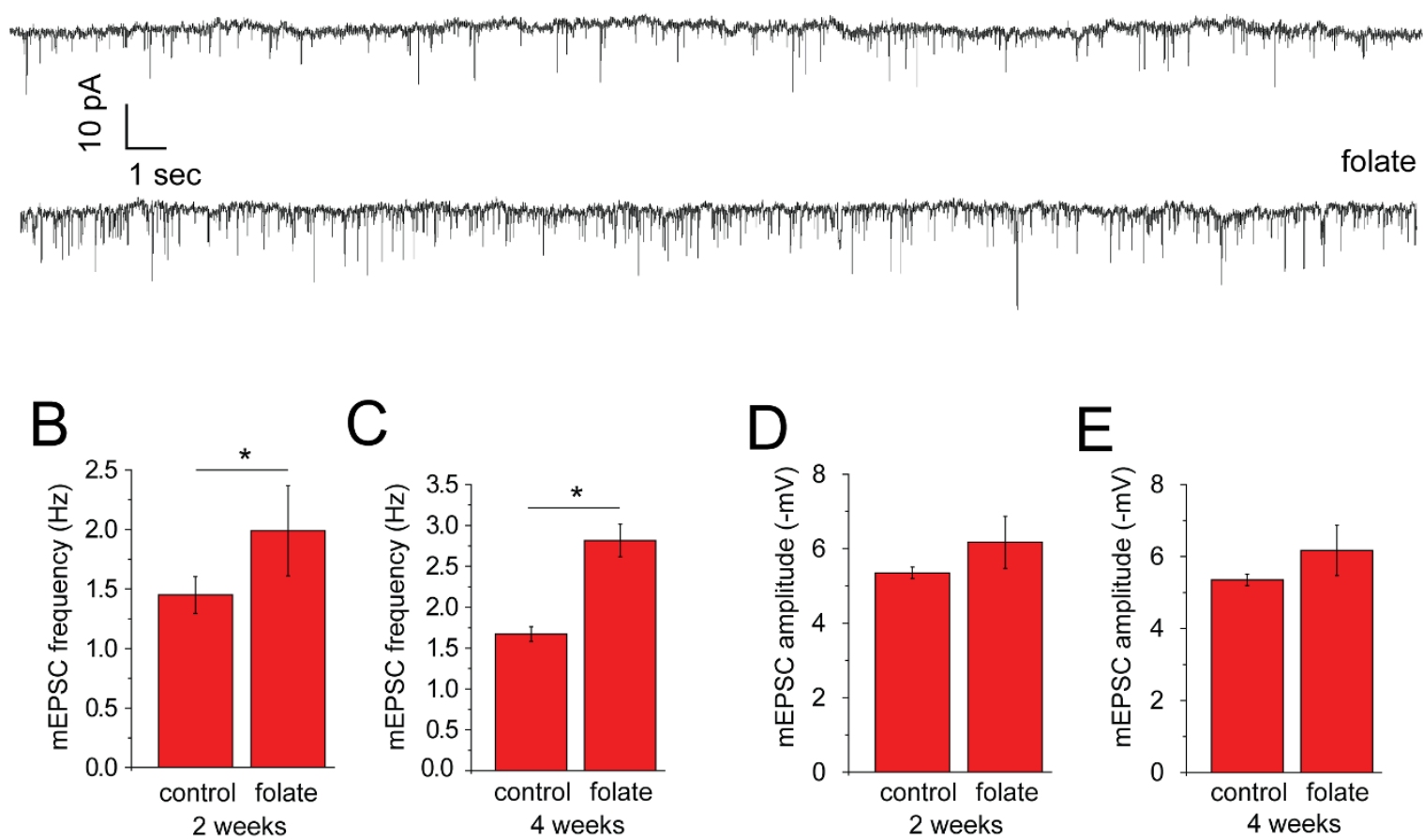

Figure 5 Miniature EPSC analysis. (A) mEPSC recorded (2 uM TTX, $-60 \mathrm{mV}$ voltage clamp) from either 2 week or 4 week old hippocampal cultures from control and folate reared conditions were analyzed to determine (B, C) average frequency and (D, E) average amplitude. Statistical differences $(*)$ were only seen for frequency $(\mathrm{n}=6$ recordings for each condition, between $50-500 \mathrm{mEPSCs}$ per recording, $\mathrm{p}<0.05)$. 
assay we found that intake of folic acid by pregnant rats resulted in offspring which had seizures twice as fast as controls when challenged by a condition which destabilized their neural activity. While this assay provides a good first indication of the predisposition of the animals to seizures, more in depth analysis of direct brain activity recordings, and their performance in more sensitive assays such as kindling will help define the differences in the offspring exposed to folic acid in utero. It should also be noted that the dose given to the pregnant rats $(4 \mathrm{mg} /$ day) is difficult to compare to and substantially higher than that typically taken by to humans $(0.4 \mathrm{mg}-$ $1 \mathrm{mg} /$ day, up to $5 \mathrm{mg}$ /day in at-risk populations), We do not hypothesize that high levels of folic acid alone are sufficient to result in offspring with a seizure disorder, but rather that it could contribute to this possibility when combined with other destabilizing factors or mutations. Consequently, higher doses were used in the in vivo assay in order to observe any resulting hyperactivity or instability in a normal rat brain background. Future studies could utilize seizureprone rats, which could more closely duplicate our hypothesized paradigm.

To further investigate this phenomenon we established an in vitro neuronal culture assay system and showed that cultures grown in the presence of $4 \mathrm{Hfolate}$ have a reduced capacity to re-regulate network activity patterns following a perturbation, a result similar in principle to the in vivo study. A reduced capacity to stabilize following a highfrequency burst could be problematic, as for the brain to perform normally it must be able to function in multiple states, including bursts of high frequency activity. It must also be able to reestablish a stable level of activity following such bursts.

Our choice of using $4 \mathrm{Hf}$ folate was primarily based on the fact that in an in vitro system there is no liver to convert folic acid to its biologically active forms and $4 \mathrm{Hfolate}$ is the highest-level metabolite past this point. Cultures were frequently replenished with the compound since its stability in solution is short, and, importantly, it is possible that the catabolites of 4 Hfolate are in fact causal to the system instability. For example it is quite plausible that the presence of higher levels of L-glutamate could be stimulating a more rapid rate of development of neuronal connectivity. However since we were uncertain of how much of the 4 Hfolate gets converted to $5 \mathrm{M} 4 \mathrm{Hfolate}$ (a very biologically relevant form) by the cells, we repeated the in vitro activity experiments with direct exposure to low levels of 5M4Hfolate. This produced an interesting result: a subset of cells became very hyperexcitable, firing at high frequency in bursts. This result demonstrates the diversity of effects that could be produced by folic acid supplementation, as it could occur through a unique mechanism. Nonetheless these experiments demonstrate that folic acid and its derivatives can alter connectivity and dynamics in a way that produces a lack of network stability or the hyperexcitability of individual neurons.

It is important to recognize that there are two layers to the mechanisms under investigation. The first relates to how exogenous folic acid causes the formation of an aberrant network during development. Given the evidence presented here this could be through a general increase in activity during development, given the fact that neuronal activity can directly regulate CNS development through homeostatic mechanisms such as synaptic scaling (for review see ${ }^{15}$ ). The $5 \mathrm{M} 4 \mathrm{H}$ folate results further suggest that cell signaling mechanisms might also be altering connectivity through internal regulatory pathways in individual neurons. The second layer to consider is what makes the folate reared network less stable or hyperexcitabile once altered. Analysis of synaptic distribution suggests not only is the overall density different, but the organization could be as well. Investigation of this, and correlation with the spatial and temporal activity patterns will hopefully lead to a better understanding of what makes a neuronal circuit epileptogenic.

The most parsimonious interpretation of this data is that, by virtue of its relationship to the excitatory neurotransmitter L-glutamate, folic acid present at continued high levels during neuronal development causes an increase in neuronal activity. This in turn accelerates the developmental of neuronal connectivity, which then leads to a hyper-excitable network. However the full spectrum of the effects of folic acid and its specific metabolites continues to be investigated. As a DNA methylating agent, folic acid is a candidate for inducing epigenetic changes, an interesting factor to consider given the novel presentation of epilepsy in families with no genetic pre-disposition. However a full understanding of these mechanisms is crucial, as previously unrealized advantages of full term folic acid supplementation might exist. For instance, recent studies have suggested a link between continued folic acid supplementation and reduced incidences of autism ${ }^{16}$. To reiterate, the presence of folic acid during the first trimester is absolutely essential, and the goal of this study is not to make a clinical recommendation for maternal health. Rather we hope this study encourages further investigation of the necessity and consequences of continued folic acid supplementation in late gestation.

\section{Methods}

In vivo assay. Young sexually mature female Long-Evans rats were paired with age matched males and given daily oral supplements of $4.0 \mathrm{mg}$ folic acid in $1 \mathrm{ml}$ of a $10 \%$ sucrose solution, while controls were given sucrose alone. After parturition the oral folic acid was discontinued and the litters culled to 6 male pups. At postnatal day 14 their latency to first behavioral seizure (clonus) following an i.p. injection of $15 \mathrm{mg} / \mathrm{kg}$ kainic acid (KA) was determined. This latency to the first behavioral seizure after KA administration, referred to as the seizure onset time (SOT), was defined by the occurrence of forelimb clonus, rearing, and loss of balance. Seizure onset time is a commonly used measure to describe susceptibility to convulsant compounds in rats ${ }^{17}$ and is related to clear paroxysmal epileptiform discharges ${ }^{18}$. Pups were sacrificed humanely with a pentobarbital overdose at the end of a one hour observation period. One pup in the control group did not display a seizure and was removed from the subsequent analysis.

In vitro assay. To investigate the cellular- and network-level effects of long term folic acid exposure, we used an in vitro model system based on dissociated cultures of rat hippocampal neurons. Briefly, high density co-cultures of neurons and glia were produced from P0 pups from normal Sprague Dawley rats as previously described ${ }^{19}$ Cultures were grown on silicon wafers to facilitate the firing of sub-populations of neurons at a precise frequency using photoconductive stimulation. Briefly, the technique works by selectively illuminating the region of the wafer to be stimulated, which increases the conductivity of the underlying silicon due to the photoconductive effect. Then a short $(2 \mathrm{~ms})$ pulse is applied across the surface of the wafer which initiates action potentials in the neurons within the illuminated region ${ }^{20}$. Experimental cultures received $4 \mathrm{Hfolate}$ or $5 \mathrm{M} 4 \mathrm{Hf}$ folate supplements daily in their growth media at a concentration of $50 \mathrm{nM}$. Supplements were prepared by dissolving the appropriate amount of the compound in media (BME) to make an $11 \mu \mathrm{m}$ stock solution. This stock solution became diluted to $50 \mathrm{nM}$ upon addition to the active culture media. The concentration chosen was estimated based on measures of actual folate blood levels ${ }^{21}$ Using this paradigm, we tested if the presence of folate acid metabolites during development causes a change in the activity patterns of the neuronal networks that develop. To quantify this, we first determined spontaneous network activity during a two-minute baseline period. Briefly, neuronal activity was recorded by Fluo- 4 calcium imaging under low magnification over a large $(\sim 100 \mathrm{~mm}$ diameter) region of the neuronal culture with a high sensitivity camera. Optical data was recorded at $33 \mathrm{~ms}$ intervals, individual cells identified, their intensity traced over time and action potentials identified using custom software written in Matlab.

Immunocytochemistry and synaptic density analysis. Neuronal cultures were fixed in $4 \%$ paraformaldehyde and $15 \%$ picric acid prepared in PBS, and then subjected to immunofluorescent labeling with an mouse monoclonal anti-synaptophysin antibody (Sigma-Aldrich, St. Louis). Images were taken with a $20 \times$ water immersion objective and processed with a Richardson-Lucy algorithim using the DeconvolutionLab plugin (Biomedical Imaging Group, EPFL) for ImageJ (NIH). The deconvoluted images were analyzed with the Graylevel Watershed (Biomedical Imaging Group, EPFL) plugin for ImageJ, which compartmentalized and counted the individual synapses in the image.

Electrophysiology. Whole-cell patch-clamp recordings were performed using an Axon Multiclamp $700 \mathrm{~b}$ amplifier, 1440A Digidata digitizer and pClamp software (Axon Instruments, Foster City, CA). Recordings were made at $50 \mathrm{kHz}$, and subsequently filtered at $5 \mathrm{kHz}$. Extracellular bath solution (EBS) for the recordings contained $135 \mathrm{mM} \mathrm{NaCl}, 10 \mathrm{mM}$ glucose, $3 \mathrm{mM} \mathrm{CaCl}_{2}, 5 \mathrm{mM} \mathrm{KCl}, 2 \mathrm{mM} \mathrm{MgCl}_{2}$ and $5 \mathrm{mM}$ Hepes, $\mathrm{pH}$ adjusted to 7.3 with $\mathrm{NaOH}$ and $310 \mathrm{mOsm}$ with Sorbital. Patch pipettes were pulled from borosilicate GD-1 glass capillaries (Narishige Scientific, Japan) and filled with intracellular solution containing $100 \mathrm{mM} \mathrm{K}$-gluconate, $1.7 \mathrm{mM} \mathrm{KCl}, 0.6 \mathrm{mM}$ EGTA, $5 \mathrm{mM} \mathrm{MgCl}_{2}, 10 \mathrm{mM}$ Hepes, $4 \mathrm{mM}$ ATP and $0.1 \mathrm{mM}$ 
GTP, adjusted to $\mathrm{pH} 7.2$ with $\mathrm{NaOH}$ and 300 mOsm with glucose. Neurons with a resting membrane potential of less than $-45 \mathrm{mV}$ were not used for our analysis. Once a neuron was patched, the seal was monitored and if the leak current potential was greater than $80 \mathrm{pA}$, the recording was not used for analysis. Miniature EPSCs (mEPSCs) recordings were made holding the potential at $-60 \mathrm{mV}$ in the presence of $1 \mathrm{uM}$ of tetrodotoxin (TTX) (Sigma-Aldrich, St. Louis), which was used to block action potentials. Action potentials were recorded in current clamp mode, $\mathrm{I}=0$. mEPSCs were identified using the template match search (pClamp) and basic statistical analysis performed to extract average amplitude and frequency. Results are presented as mean \pm SEM throughout the text unless otherwise noted. The term significant denotes a relationship with $\mathrm{p}<0.05$ determined using an unpaired Student's t-test.

Use of animals. All experimental protocols were approved by the University of Calgary Conjoint Faculties Research Ethics Board, and performed under ACC protocol \#AC12-0242.

1. Smithells, R. W., Sheppard, S. \& Schorah, C. J. Vitamin dificiencies and neural tube defects. Arch Dis Child 51, 944-950 (1976).

2. Boyles, A. L. et al. Neural tube defects and folate pathway genes: family-based association tests of gene-gene and gene-environment interactions. Environ Health Perspect 114, 1547-1552 (2006).

3. O'Rahilly, R. \& Muller, F. Developmental stages in human embryos: revised and new measurements. Cells Tissues Organs 192, 73-84 (2010).

4. Pickell, L. et al. High intake of folic acid disrupts embryonic development in mice. Birth Defects Res A Clin Mol Teratol 91, 8-19 (2011).

5. Kehl, S. J., McLennan, H. \& Collingridge, G. L. Effects of folic and kainic acids on synaptic responses of hippocampal neurones. Neuroscience 11, 111-124 (1984).

6. Otis, L. C., Madison, D. V. \& Nicoll, R. A. Folic acid has a disinhibitory action in the rat hippocampal slice preparation. Brain Res 346, 281-286 (1985).

7. Stephens, R. L. Jr. \& Uretsky, N. J. Folate induced-hypermotility response after bilateral injection into the nucleus accumbens of the rat. Possible mediation through dopaminergic mechanisms. Neuropharmacology 25, 887-896 (1986).

8. Khazipov, R. et al. Early development of neuronal activity in the primate hippocampus in utero. J Neurosci 21, 9770-9781 (2001).

9. Ichi, S. et al. Fetal neural tube stem cells from Pax3 mutant mice proliferate, differentiate, and form synaptic connections when stimulated with folic acid Stem Cells Dev 21, 321-330 (2012).

10. Stanger, O. Physiology of folic acid in health and disease. Curr Drug Metab 3, 211-223 (2002).

11. Hyland, K., Shoffner, J. \& Heales, S. J. Cerebral folate deficiency. J Inherit Metab Dis 33, 563-570 (2010)

12. Fava, M. \& Mischoulon, D. Folate in depression: efficacy, safety, differences in formulations, and clinical issues. J Clin Psychiatry 70 Suppl 5, 12-17 (2009).

13. Collin, S. M. et al. Association of folate-pathway gene polymorphisms with the risk of prostate cancer: a population-based nested case-control study, systematic review, and meta-analysis. Cancer Epidemiol Biomarkers Prev 18, 2528-2539 (2009)
14. Prasad, A. N., Rupar, C. A. \& Prasad, C. Methylenetetrahydrofolate reductase (MTHFR) deficiency and infantile epilepsy. Brain Dev 33, 758-69 (2011).

15. Turrigiano, G. G. The self-tuning neuron: synaptic scaling of excitatory synapses Cell 135, 422-435 (2008).

16. Schmidt, R. J. et al. Maternal periconceptional folic acid intake and risk of autism spectrum disorders and developmental delay in the CHARGE (CHildhood Autism Risks from Genetics and Environment) case-control study. Am JClin Nutr 96, 80-89 (2012).

17. Galic, M. A. et al. Postnatal inflammation increases seizure susceptibility in adult rats. J Neurosci 28, 6904-6913 (2008).

18. Pitkèanen, A., Schwartzkroin, P. A., Moshâe, S. L. \& ebrary, I. Models of seizures and epilepsy (Elsevier Academic, Amsterdam, Boston, 2006).

19. Colicos, M. A., Collins, B. E., Sailor, M. J. \& Goda, Y. Remodeling of synaptic actin induced by photoconductive stimulation. Cell 107, 605-616 (2001).

20. Goda, Y. \& Colicos, M. A. Photoconductive stimulation of neurons cultured on silicon wafers. Nat Protoc 1, 461-467 (2006).

21. Tamura, T. \& Picciano, M. F. Folate and human reproduction. Am J Clin Nutr 83, 993-1016 (2006).

22. Moddemeijer, R. On estimation of entropy and mutual information of continuous distributions. Signal Processing 16, 233-248 (1989)

\section{Acknowledgments}

The authors would like to thank Robyn Flynn for critical reading of the manuscript. This research was funded by grants from CIHR to MAC, CIHR and NSERC to GCT and AIF to JD, and the Thomas Tyson Endowment for Research in Epilepsy to MC and GCT.

\section{Author contributions}

M.C. authored the main manuscript text and prepared the figures; F.G. and J.D. designed and performed the in vitro activity pattern and complexity analysis, L.S. performed the long term in vitro experiments, Y.A. performed the mEPSC experiments, J.H. initiated and performed a preliminary study, S.I. performed the synaptic density experiments, C.D.M. investigated the acute folate electrophysiology, R.T. assisted with protein chemistry, L.B.E. provided the initial incentive for the study, J.R. advised on clinical relevance, C.T. administered the in vivo latency to seizure assay. All authors reviewed the manuscript.

\section{Additional information}

Competing financial interests: The authors declare no competing financial interests.

License: This work is licensed under a Creative Common

Attribution-NonCommercial-NoDerivs 3.0 Unported License. To view a copy of this license, visit http://creativecommons.org/licenses/by-nc-nd/3.0/

How to cite this article: Girotto, F. et al. High dose folic acid supplementation of rats alter synaptic transmission and seizure susceptibility in offspring. Sci. Rep. 3, 1465; DOI:10.1038/ srep01465 (2013). 